\title{
Transformación educativa mediada con tecnología digital: oportunidad única de cara a la "nueva normalidad"
}

\author{
Educational transformation mediated with digital technology: \\ a unique opportunity facing the "new normal" \\ Transformação educacional mediada com tecnologia digital: \\ oportunidade única diante do "novo normal"
}

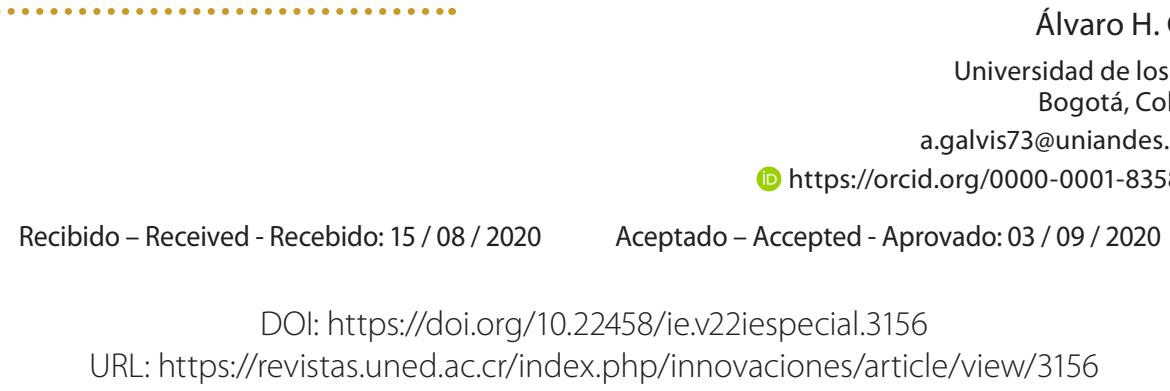

\begin{abstract}
Resumen: La pandemia que estamos viviendo ha incrementado la velocidad de cambio en educación a todos los niveles, al menos en lo que se refiere a utilización de tecnologías digitales (TD) para apoyar procesos educativos y de gestión de la misma; en pocos días la gran mayoría de docentes y estudiantes pasamos de interactuar en aulas físicas a hacerlo en forma remota. Una pregunta fundamental que nos venimos haciendo al estar a punto de volver a una "nueva normalidad" es ¿cómo se puede aprovechar la coyuntura para propiciar transformación educativa mediada con TD?, y su corolario, ¿qué hay que cuidar en especial para tener éxito en esto? Este editorial científico sustenta que dicha transformación educativa es posible y exige cuidar las dimensiones educativa, tecnológica, organizacional y cultural, que son consustanciales a los procesos de cambio en organizaciones educativas
\end{abstract}

Palabras clave: Ambientes de aprendizaje; autonomía y autocontrol en procesos educativos; entornos de aprendizaje; TD para enriquecer la educación; transformación educativa mediada con TD, COVID-19

\begin{abstract}
The pandemic that we are experiencing has increased the speed of change in education at all levels, at least with regard to the use of digital technologies (DT) to support educational processes and education management. In a few days, the vast majority of teachers and students went from interacting in physical classrooms to doing it remotely. A fundamental question that we have been asking ourselves when we are about to return to a "new normal" is how can the situation be taken advantage of to promote educational transformation mediated with DT?, and its corollary, what must be especially taken care of to be successful in this? This scientific editorial maintains that said educational transformation is possible and requires taking care of the educational, technological, organizational and cultural dimensions, which are inherent to the processes of change in educational organizations.
\end{abstract}

Key Words: Learning environments; autonomy and self-control in educational processes; learning environments; DT to enrich educati, COVID-19 
Resumo: A pandemia que estamos vivendo aumentou a velocidade da mudança na educação em todos os níveis, pelo menos em termos do uso de tecnologias digitais (DT) para apoiar processos educacionais e de gestão; em poucos dias, a grande maioria dos professores e alunos passou de interagir em salas de aula físicas para fazêlo de forma remota. Uma pergunta fundamental que temos feito a nós mesmos enquanto estamos prestes a retornar a uma "nova normalidade" é como podemos aproveitar a situação para promover a transformação educacional através da tecnologia digital, e seu corolário, o que deve ser cuidado, em particular, para ter sucesso nisso? Este editorial científico argumenta que tal transformação educacional é possível e requer o cuidado com as dimensões educacional, tecnológica, organizacional e cultural, que são inerentes aos processos de mudança nas organizações educacionais.

Palavras-chave: Ambientes de aprendizagem; autonomia e autocontrole nos processos educacionais; ambientes de aprendizagem; TD para enriquecer a educação; TD - transformação educacional mediada por TD, COVID-19

\section{INTRODUCCIÓN}

En las últimas décadas, ha habido transformación educativa con apoyo de TD a distintos niveles del sector educativo, en distintas disciplinas y haciendo uso de diversidad de modalidades educativas, sin que esto sea generalizado. Los cambios suelen ser promovidos por docentes o directivos innovadores, que se atreven a someter a prueba estrategias pedagógicas promisorias o que se entusiasman con las oportunidades educativas que hallan en las TD. Por lo general, esto se da dentro de ecologías para el aprendizaje que favorecen dichas prácticas, pues ha habido esfuerzos importantes por dotar a los miembros de las distintas comunidades educativas de computadores conectados a internet, con acompañamiento a los procesos de cambio educativo, con miras a propiciar cultura digital que apoye los modelos educativo y operativo con los que haya compromiso institucional o, cuando menos, por programa. Lo que sucedió a raíz de la pandemia desencadenada por el virus responsable del COVID 19, sin embargo, no es necesariamente escalamiento de dichas iniciativas, sino un "tener que usar TD" para superar las dificultades de interacción en campus físicos entre docentes, estudiantes y demás miembros de cada comunidad educativa (administrativos, padres de familia, empleadores, grupos de interés). Cada educador, dependiendo de su propia trayectoria y de los niveles de aprestamiento tecnológico de su organización y propio, se ha visto en la necesidad de atender de la mejor manera posible el reto de pasar a la enseñanza remota, haciendo uso de su mejor estilo docente y de las posibilidades que brindan TD a su disposición. Muchas organizaciones educativas han dado pasos rápidos y poderosos para ayudar a sus miembros a tener acceso y lidiar con entornos de enseñanza y aprendizaje mediados con TD, en contextos familiares donde se comparten espacios y acceso a TD a lo largo del día. También se ha tenido que prestar atención a factores emocionales, de salud física y mental, de inestabilidad laboral y hasta de inseguridad alimentaria. La opinión de muchos, incluido yo, es que en tres o cuatro meses se dio un salto predominantemente cuántico en uso generalizado de TD para educación (más de lo que se solía hacer con internet, poco de innovación educativa), quedando pendiente la posible transformación educativa de procesos y programas que lo ameriten.

Parece ser que, de seguir con el debido autocuidado y medidas de sanidad individual y colectiva, se podrá retornar al uso del campus físico y armonizarlo con lo que se puede haber ido conformando como campus virtual en cada organización educativa, combinación a la que se suele llamar la "nueva normalidad", concepto que aún está en construcción. Las preguntas que surgen en este escenario de cambio sobre la marcha incluyen: ¿en qué dirección hacer transformaciones educativas para enriquecer una nueva realidad social?, ¿cómo hacer transformación educativa apoyada en TD en época de pandemia?, ¿qué hay que hacer muy bien para tener éxito en este proceso? 


\section{TRANSFORMACIÓN EDUCATIVA EN ÉPOCA DE ENSEÑANZA BÁSICAMENTE NO PRESENCIAL}

En cuanto a la dimensión educativa, se acepta que la transformación educativa con apoyo de TD tiene que ver con cambios en el eje de control del proceso de enseñanza-aprendizaje, de modo que haya autonomía creciente en el estudiante para la gestión del conocimiento, en interacción con compañeros y docentes, aprovechando las oportunidades que brindan las TD para flexibilizar los entornos, ambientes y los medios para aprender (Boekaerts, 1997; Zimmerman \& Schunk, 2008; Galvis Á. H., 2010; Bates, 2015), lo cual conlleva cambios en concepciones, prácticas y herramientas para educar (Salinas, 2004).

Esto es posible gracias a la confluencia de ideas educativas transformadoras, como que la educación es mucho más que escolaridad o prepararse para una vida productiva, la educación es vida (Lindeman, 1926); que el aprendizaje y la enseñanza, si bien están interrelacionados, requieren privilegiar que el aprendiz sea cada vez más autónomo y parte de comunidades que aprenden (Cognition and Technology Group at Vanderbilt, 1998; Wenger, 1998; Ambrose, Bridges, DiPrietro, Lovettt, \& Norman, 2010); esto genera retos al docente para que sea diseñador de ambientes de aprendizaje que tomen en cuenta al aprendiz, lo disciplinar, lo formativo y lo comunitario (Bransford, Brown, \& Cocking, 2000) y que crecientemente su rol sea como facilitador de interacciones educativas (Collison, Elbaum, Haavind, \& Tinker, 2000).

Desde el punto de vista de los medios digitales, la transformación mencionada lleva a reconocer la coexistencia y rol propio de tres tipos de medios complementarios: los de tipo interactivo, con los se aprende por interacción con otros; los de tipo activo, donde se aprende por acción del sujeto sobre objetos digitales con comportamiento orgánico; y los de carácter expositivo, con los que se aprende por transmisión de modelos mentales encapsulados por su autor para uso del aprendiz a ritmo y en secuencia propia (Forté \& Wentland, 1998), dentro de estructuras de aprendizaje flexibles (que pueden adaptarse según lo amerite el comportamiento del aprendiz) y con distintos niveles de inteligencia (el sistema apoya toma de decisiones con base en información acerca del aprendiz y de su desempeño individual o como parte de redes de aprendizaje, así como en las intenciones que mueven el proceso) a partir de análisis de distintos tipos de datos (Gros, 2016; Bartolomé, Castañeda, \& Jordi, 2018).

Lo anterior sirve de base para afirmar, con Graham (2020), que en la transición de una modalidad básicamente presencial a otra básicamente no presencial (virtual o híbrida) como es la que ha favorecido la pandemia, lo que hace diferencia es el modo como se diseña y lleva a cabo el proceso de enseñanzaaprendizaje, antes que la modalidad misma. Sostiene dicho autor que, en cualquier modalidad, es necesario cuidar tanto el componente afectivo (haciendo sentir en comunidad a los participantes) como el comportamental (ligado a fomentar hábitos de estudio crecientemente autónomos y a desarrollar autocontrol para cumplir con los compromisos de estudiar); así mismo, destaca la importancia de diseñar y acompañar el componente cognitivo, para lograr entendimiento y aplicación de los conceptos disciplinares fundamentales, bajo distintas circunstancias, lo más cercanas posible a lo esperado para demostrar el nivel de competencia deseado.

Lo anterior no significa que el conocimiento que tenemos acerca de cómo transformar ambientes educativos en modalidad básicamente presencial se transfiera directamente a la puesta a punto de ambientes virtuales o híbridos transformados. Estudios de buenas prácticas en educación superior en modalidad virtual o híbrida (Galvis Á. H., 2018; Galvis Á. H., 2019) muestran que el componente organizacional hace diferencia, siendo clave el compromiso directivo con la utilización de la o las modalidades no presenciales que se escojan, pues esto alinea la acción institucional a todo lo largo de la cadena de valor y de soporte; así mismo, muestran que el modelo educativo que se adopte para lo sustantivo debe ser mucho más que una prédica; no basta con declarar que, por ejemplo, se comparten principios socioconstructivistas para la puesta a punto de los ambientes de aprendizaje, sino que hay que lograr que la comunidad educativa los viva y enriquezca a partir de la reflexión sobre los programas educativos que 
se diseñan, sobre los cursos y los materiales que se ponen a punto, sobre los sistemas de evaluación que se utilizan, así como para la preparación del recurso humano que acompaña a los estudiantes en su proceso, sean directores de programa o de curso, tutores disciplinares o consejeros, se impone alineación de todos con el o los modelos educativos y operativos que se adopten.

Lo tecnológico también juega un rol importante, pues la flexibilidad curricular y pedagógica en entornos de aprendizaje virtuales, personales o grupales, requiere mucho más que acceso a tecnología, lo cual es una condición necesaria; la combinación de medios debe apalancar las estrategias pedagógicas que hacen realidad el diseño educativo, deben hacer posible que la evaluación sea educativa, que lo inteligente de los sistemas de análisis de información y de adaptabilidad de los sistemas de enseñanza y aprendizaje sean un potencial puesto al servicio de los procesos educativos.

No menos importante es lo cultural en las múltiples dimensiones mencionadas. Las viejas prácticas ligadas a procesos presenciales que dan soporte a la cadena de valor requieren procesos en entornos virtuales que sean inteligentes e intuitivos, con robustez y oportunidad cuando se decide poner a punto un campus virtual, el cual se espera que cumpla al menos con las mismas funciones del campus físico. Esto va más allá de contar con la tecnología y los procedimientos a punto para la experiencia virtual, requiere cambio de mentalidad entre quienes intervienen en los distintos procesos, sean estos sustantivos (macro-, meso- y micro-curriculares) o de soporte a lo largo de la cadena de valor (desde los estudios de necesidades y conocimiento de los grupos de interés, hasta la certificación / graduación, y oferta continuada de oportunidades en atención a las necesidades cambiantes).

\section{DE CARA A LA “NUEVA NORMALIDAD”}

Mencioné antes que "nueva normalidad" no es un concepto bien definido y, por eso, precisamente, es muy interesante y retador. La pandemia ha obligado a repensar nuestros conceptos acerca de lo que significa ofrecer educación superior a quienes, por ahora y no se sabe por cuánto más tiempo, tendrán que participar en forma remota y desde sus hogares, o intermitente presencial / remota, en procesos educativos -y laborales- de su incumbencia. También ha obligado a analizar las prácticas y herramientas educativas actuales, en cualquier modalidad que ellas se lleven a cabo, pues la coyuntura ha puesto de presente la necesidad de apalancar en el estudiantado autonomía y autocontrol creciente en procesos educativos, al tiempo que las organizaciones educativas están llamadas a ser flexibles, pertinente e innovadoras, como organizaciones que aprenden en su diálogo con el entorno y con los actores del proceso. Tenemos a disposición una cantidad de ideas y oportunidades poderosas. Se debe buscar la manera de hacerlas realidad y salir fortalecidos de la pandemia.

Nota: La página web del autor se encuentra en https://academia.uniandes.edu.co/AcademyCv/a.galvis73 
Ambrose, S., Bridges, M., DiPrietro, M., Lovettt, M. y Norman, M. (2010). How Learning Works: Seven Research-based Principles for Smart Teaching. San Francisco, CA: Jossey-Bass.

Bartolomé, A., Castañeda, L. y Jordi, A. (2018). Personalisation in educational technology: the absence of underlying pedagogies. International Journal of Educational Technology in Higher Education, 15(14), 2-17. doi:10.1186/s41239-018-0095-0

Bates, A. (2015). Teaching in a digital age. Guidelines for designing teaching and learning. Recuperado de https://opentextbc.ca/teachinginadigitalage/

Boekaerts, M. (1997). Self-regulated learning: A new concept embraced by researcher, policy makers, educators, teachers, and students. Learning and Instruction, 7(2), 161-186. doi:10.1016/ S0959-4752(96)00015-1

Bransford, J., Brown, A. y Cocking, R. (2000). The design of learning environments. En J. Bransford., A. Brown, y R. Cocking (Edits.), How People Learn - Brain, mind, experience, and school (págs. 131-154). Washington, DC: National Academy Press.

Cognition and Technology Group at Vanderbilt. (1998). Designing Environments to Reveal, Support, and Expand Our Children's Potentials. En S. Soraci, y W. Mcllvane (Edits.), Perspectives on Fundamental Processes in Intellectual Functioning: A Survey of Research Approaches (págs. 313-350). Stamford, CT: Ablex.

Collison, G., Elbaum, B., Haavind, S. y Tinker, R. (2000). Facilitating online learning - Effective strategies for moderators. Madison, Wl: Atwood Publishing.

Forté, E. y Wentland, M. (1998). The ARIADNE project: knowledge pools for computer-based and telematics supported classical, open and distance education. En Forté, E. y Wentland, M. (1998). AAUC Ariadne Academic Users Group Conference (págs. 1-23). Lucerna, Switzerland: AAUC Conference.

Galvis, Á. H. (agosto, 2010). Nuevos ambientes educativos basados en tecnología. Revista Sistemas, (117). Recuperado de http://52.0.140.184/typo43/fileadmin/Revista_117/Columnista_invitado.pdf

Galvis, Á. H. (2018). Supporting decision-making processes on blended learning in higher education: literature and good practices review. International Journal of Educational Technology in Higher Education, 15(25), 1-38. doi:10.1186/s41239-018-0106-1

Galvis, Á. H. (2019). Direccionamiento estratégico de la modalidad híbrida en educación superior: Conceptos, métodos y casos para apoyar toma de decisiones. Bogotá: Ediciones Uniandes.

Graham, C. R. [Viceministerio Educación Preescolar, Básica y Media]. (28 de julio de 2020). Educ@TeD 2020 (Día 1) [Archivo de video]. Recuperado de https://youtu.be/kdcdtQM776o

Gros, B. (2016). The design of smart educational environments. Smart learning environments, 3(15), 2-17. doi:10.1186/s40561-016-0039-x

Lindeman, E. (1926). The meaning of Adult Education. New York: New Republic.

Salinas, J. (2004). Innovación docente y uso de las TIC en la enseñanza universitaria. Revista Universidad y Sociedad del Conocimiento (RUSC), 1(1), 1-16. doi:10.7238/rusc.v1i1.228

Wenger, E. (1998). Communities of practice: Learning, meaning, and identity. Cambridge,: Cambrige University Press. doi:10.1017/СBO9780511803932

Zimmerman, B. y Schunk, D. (2008). Motivation: An essential dimension of self-regulated learning. En D. Schunk, \& B. Zimmerman (Edits.), Motivation and self-regulated learning: Theory, research, and applications (págs. 1-30). Mahwah, NJ: Lawrence Erlbaum. doi:10.4324/9780203831076 